\title{
Size scaling with light patterned dielectrophoresis in an Optoelectronic Tweezers device
}

\author{
Angel Fuentes ${ }^{\mathrm{b}}$, Juan Carlos Rodriguez Luna ${ }^{\mathrm{a}}$, Joan Juvert ${ }^{\mathrm{a}}$, Ruben Ramos-Garcia ${ }^{\mathrm{b}}$, \\ Steven L. Neale \\ ${ }^{a}$ School of Engineering, University of Glasgow, Glasgow, G12 8LT, UK. \\ ${ }^{\mathrm{b}}$ Optics Department, Instituto Nacional de Astrofísica, Óptica y Electrónica Luis Enrique Erro \#1 \\ Sta. Ma. Tonantzintla, 72840 Puebla, Mexico.
}

\begin{abstract}
We report the experimental measurement of the relationship between the size of particles being moved by optically patterned dielectrophoresis in an Optoelectronic Tweezers (OET) device and the force that they experience. The OET device turns an optical pattern into a pattern of electrical fields through the selective illumination of a photoconductive material. In this work we use a data projector to create the structured illumination which gives a relatively flat optical profile with steep optical gradients and hence steep electrical gradients at the edges of the light patterns created. For a small particle in a constant electrical gradient it would be expected that the force due to dielectrophoresis would scale with the cube of the particle's radius whereas the forces needed to move it against the viscous fluid scale with the radius so that there would be a an increase of the velocity at which we can move particles with a relationship of the radius squared. As the particles in an OET device are often larger than the area over which the electrical gradients are produced it is not obvious how their forces scale with size. In this paper we show that there is a small size regime where the particle size relationship with force is well described by a linear fit and a regime where it is not. We show that the magnitude of the force is dependent on the light pattern used and that with larger particles and optimized light patterns velocities of around $1 \mathrm{mms}^{-1}$ can be achieved.
\end{abstract}

Keywords: optoelectronic tweezers, dielectrophoresis, optical micromanipulation

\section{INTRODUCTION}

Optoelectronic Tweezers (OET) use a light patterned photoconductive device to produce a reconfigurable dielectrophoresis force for microparticle manipulation. They have become a popular tool to manipulate objects in the micro- and nano- scale. Their popularity is due to the many advantages over the classical optical tweezers and other manipulation tools. Some of the advantages are the low optical power needed to generate the traps, the ability to perform parallel manipulation of objects and the wide range of particle size they can handle. The particle manipulation is achieved by projecting light patterns onto an amorphous silicon layer to produce virtual electrodes [1].

This powerful technique has been employed in many fields, for example in cell biology for a non-invasive manipulation of biological material [2] and for the fabrication and assembly of micro- and nano-electronic components. Some interesting works using OET have been performed in recent years, for example, separation of red (diameter: $6.2 \mu \mathrm{m}-$ $8.2 \mu \mathrm{m})$ and white cells $(10 \mu \mathrm{m}-30 \mu \mathrm{m})$ [3], the manipulation of sperms to improve assisted reproductive technologies $(3.1 \mu \mathrm{m}-5.1 \mu \mathrm{m})$ [4], Jurkat cells $(11.5 \mu \mathrm{m})$ and HeLa cells $(20 \mu \mathrm{m})$ [5], manipulation of oocytes $(126 \mu \mathrm{m}-163 \mu \mathrm{m})[6]$; and the assembly of carbon nanotubes (20nm-100nm) whit high precision [7,8].

As we can see, in the examples above there is a range of four orders of magnitude between the smaller and the larger objects that were moved with OET. It is evident that the size of the object being manipulated plays a very important roll to correctly characterize the manipulation process. The size of the object can modify the speed of manipulation which is an important parameter of if this technique is to be used in massive fabrication of nano/micro-electronic components.

The trap profiles created in an OET device have been well characterised for HeLa cells [9] however this profile is a combination of the device geometry and the physical properties of the particles being manipulated and so will vary when the particles being manipulated change. It was found that a good approximation of an ideal "Hook like" trap could be 
achieved, that is one where the trap stiffness is constant, by using a light pattern with a similar size to the cells. Larger light patterns did not increase the trap stiffness but instead introduced an area in the centre of the trap where the particle did not experience any force and hence reduced the positional control over the particle.

Despite their popularity and their advantages, there are no studies of the relation between the force that the OET uses to manipulate particles and the size of the particle being manipulated. Our work shows the experimental and numerical results of how this relationship is given inside OET, spherical particles were used to ensure that the shape of the objects would not produce any other effects on the force. There is evidence that shows that these devices work in at least two regimes, these regimes depend on the sizes of the particle being manipulated.

\section{EXPERIMENTAL}

OET uses a light patterned photoconductive device to produce patterns of electrical field. A photoconductive film forms one element of a circuit created by sandwiching a liquid sample between two conductive plates (see figure 1) one of which is coated in the photoconductor, in these experiments this is a $1 \mu \mathrm{m}$ thick hydrogenated amorphous silicon layer a$\mathrm{Si}: \mathrm{H}$. An external electrical field is then placed across the conductive plates and so this forms a capacitor like structure with a constant field within the liquid sample. When a light pattern is then focused onto the photoconductor the resistance of this film drops in this local area which causes the electrical field to become concentrated in the liquid above the illuminated a-Si. The electrical gradients that this create in the liquid can then be used to move particles through the dielectrophoresis force [10] which is the electrical gradient force that any charge neutral but polarisable particle will experience. Then by changing the light pattern the pattern of electrical forces can be changed giving dynamic control over the position of any particles that are sitting just above the photoconductor.

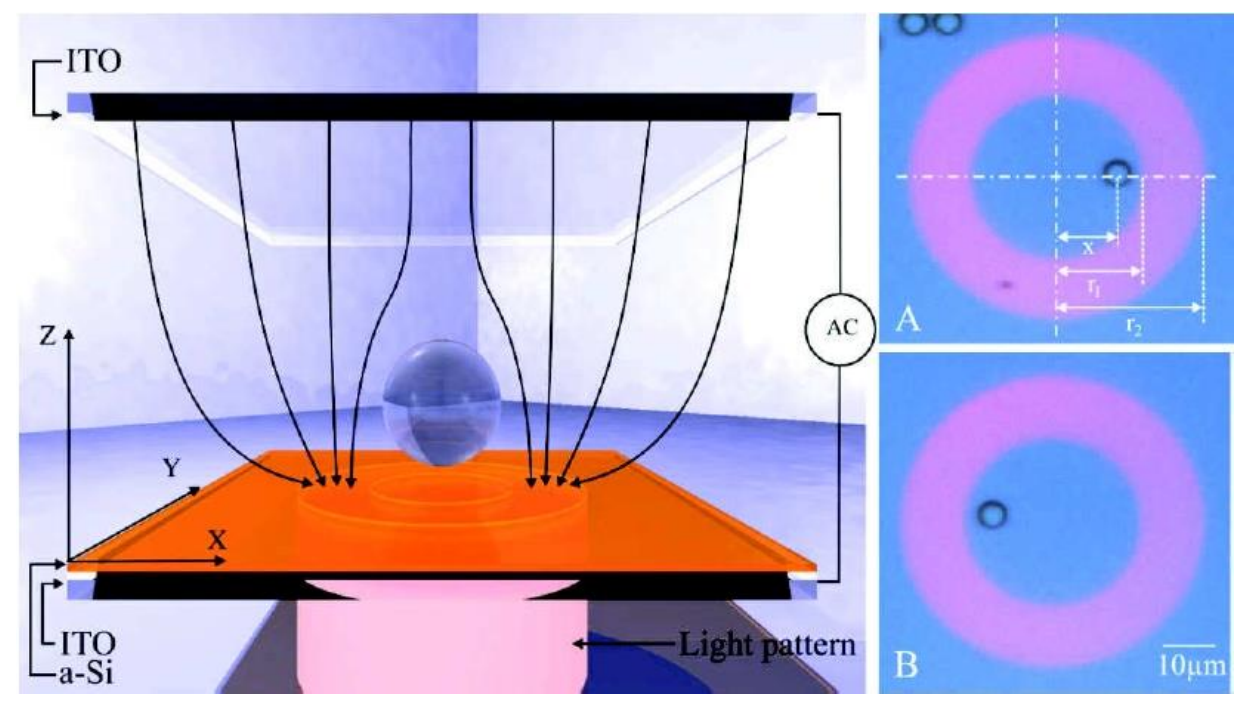

Figure 1, a schematic diagram of the OET device showing two plates coated in a transparent conductor (ITO) one of which is also coated in the photoconductive layer. A liquid sample is then sandwiched between these layers containing the colloidal particles. A) shows a ring shaped light pattern that can be used to trap particles of low polarisability that are pushed away from the high electrical field regions [11], B) when the trap is moved to the right relative to the microscope stage the particle is pushed along by the left hand edge of the light trap.

The dielectrophoretic force (DEP) is due to the difference in polarisability of the particle and the medium it is suspended in and is given by; 


$$
\vec{F}_{D E P}=2 \pi r^{3} \varepsilon_{m} \Re\left(\frac{\hat{\varepsilon}_{p}-\hat{\varepsilon}_{m}}{\hat{\varepsilon}_{p}+2 \hat{\varepsilon}_{m}}\right) \vec{\nabla} E^{2}
$$

Where $r$ is the radius of the particle, $\varepsilon_{\mathrm{m}}$ the dielectric constant of the medium, and $\varepsilon_{\mathrm{m}}$ and $\varepsilon_{\mathrm{p}}$ are the complex permittivities of the medium and the particle, respectively. The term between parentheses is the Clausius-Mossotti factor and $\mathrm{E}$ is the electrical field. If the permittivity of the particle is higher than that of the surrounding medium, the ClausiusMossotti factor is positive and the particle is attracted towards increasing field values (same direction as the gradient of the field). If the permittivity of the medium is higher, the Clausius-Mossotti factor is negative and the particle is repelled away from the regions with high electric field (direction opposite to the gradient). The Clausis-Mossotti factor varies between -0.5 and 1 for spherical particles however it can be larger for elongated particles [12].

Traditional DEP experiments involve the use of electrodes patterned onto a surface. OET have been shown to work well for moving microscopic particles with light intensities in the order of $1 \mathrm{~W} \mathrm{~cm}^{-2}$ (about 5 orders of magnitude less than conventional optical tweezers) and to produce traps that are 470x stiffer per $\mathrm{mW}$ of light used [13]. Moreover, no coherence is required, and therefore the illumination can be carried out with standard light sources, for instance a commercial projector focused onto the photoconductive surface. Since a computer can be used to send any static or moving light pattern to the projector, the potential for automation and parallelization is quite clear. There are some disadvantages to OET too and these include that the manipulation is only in 2D as we can only move particles around on the photoconductive surface plus we have to take into consideration the conductivity of the liquid when we are designing the electrical circuit which places restrictions on the buffer that we can use [14].

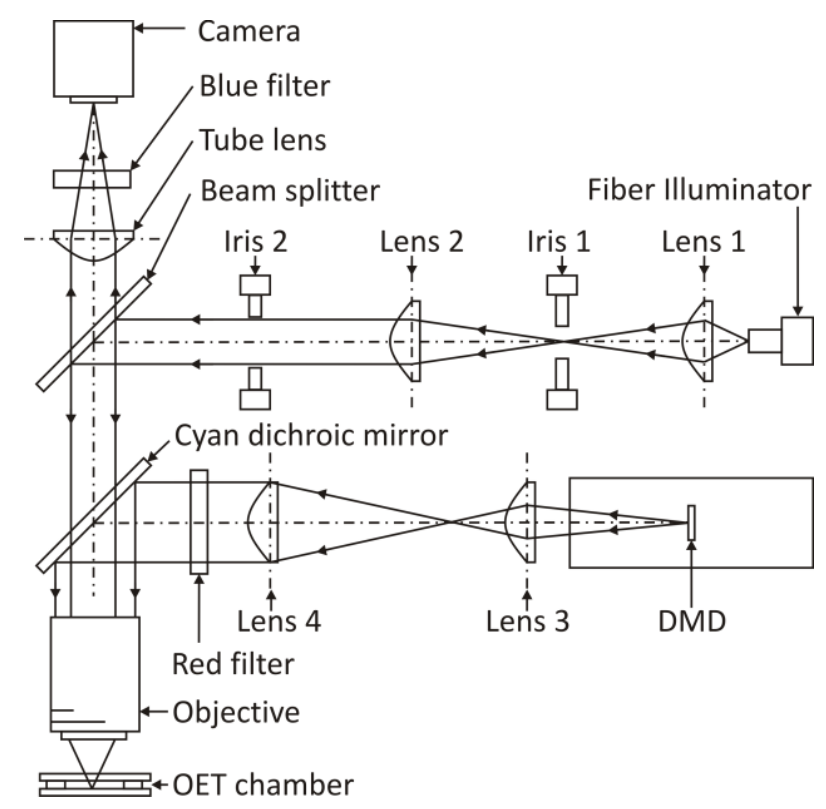

Figure 2 shows a simplified optical diagram of the optical setup used. The setup is based on an upright brightfield microscope with an extra light path for the structured illumination [15].

Figure 2 shows a simplified optical diagram of the optical setup used in these experiments. It is based on an upright brightfield microscope (Olympus, BX52) which has had an extra port fitted to allow the entry of an extra light path for the structured illumination. The extra light path is introduced into the "infinity space" of the microscope where the light rays from the infinity correct objective is roughly parallel to the optical axis and so the introduction of an extra element here does not have a great effect on the imaging path of the microscope so that the ordinary light source and camera ports can be used with no loss of optical performance. 
The particles used in this experiment were various sized glass beads (Cospheric, USA) and were suspended in buffers including pure deionised water (DI) and deionised water with added potassium chloride ( $\mathrm{KCl}$ ) to increase the conductivity of the buffer and thus change the electrical circuit in the OET device. The optimum performance of the OET device occurs when the impedance of the liquid layer in the device is in between the impedance of the dark and light areas of the photoconductor. When this condition is met then most of the voltage is placed across a-Si in the dark areas of the device and across the liquid in the light areas of the device so that there is a large electrical gradient at the edges of the light patterns.

\section{RESULTS}

The light pattern used in this work was a rotating wheel shape which could be sped up to test how fast the particles could be moved. Initial results were taken by moving a light pattern relative to the OET device in a linear direction however it was found that as the speed increased it was necessary to move the device a significant distance which made it more likely that the bead would encounter a piece of dirt and fall out of the trap. The circular spinning light pattern help as the particle can be sun repeatedly in the same area.

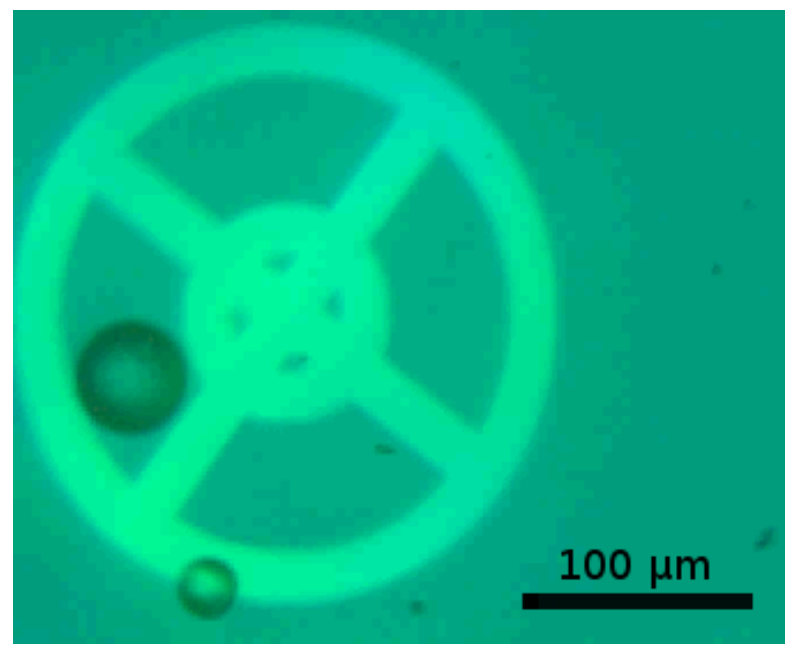

Figure 3 shows the light pattern used to move the particles at increasing speeds in order to measure what the maximum forces that it was possible to place onto the microparticles.

Figure 3 shows the light pattern that was used in these measurements. As the glass beads are insulating they are very low polarisability and so they produce a negative Clausius-Mossitti factor and thus are repelled by the high electrical field regions above the light patterns. As such the beads are pushed into the dark areas of the pattern and spun around. From the diameter of the circle that the bead describes and the speed of the rotation we can calculate the speed of the bead in micrometers per second. As the direction of the motion is constantly changing this represents a continually changing velocity and hence a constant acceleration. In micromanipulation experiments we are usually working in a regime dominated by viscous forces where inertial forces are negligible. As such accelerations are usually virtually instantaneous and so the force becomes proportional to the viscous drag as described by Stokes drag;

$$
F=6 \pi R V
$$

Where $\mathrm{F}$ is the drag force, $\mathrm{R}$ is the radius of a spherical particle and $\mathrm{V}$ is the velocity. In order to check that the viscous force does dominate in these experiment we have compared it to the centripetal force that would cause the acceleration that causes the change in direction. Centripetal force is given by; 


$$
F=\frac{m v^{2}}{r}
$$

Where $\mathrm{F}$ is the centripetal force, $\mathrm{m}$ is the mass of the particle, $\mathrm{v}$ is the tangential velocity and $\mathrm{r}$ is the radius of the circle that the particle is describing. As the mass of a particle increases with the radius cubed and the centripetal force increases with the velocity squared the highest centripetal force will be for the largest particle that we measure travelling at the highest speed we achieve. Using the 90 micron diameter bead travelling at 800 microns per second we have calculated the centripetal force as $5.9 \mathrm{pN}$ and the Stokes drag force as $0.7 \mu \mathrm{N}$ and as such we can see that the viscous forces are several orders of magnitude higher so that the speed at which we can spin particles is a good measure of how fast we can move them against drag forces.
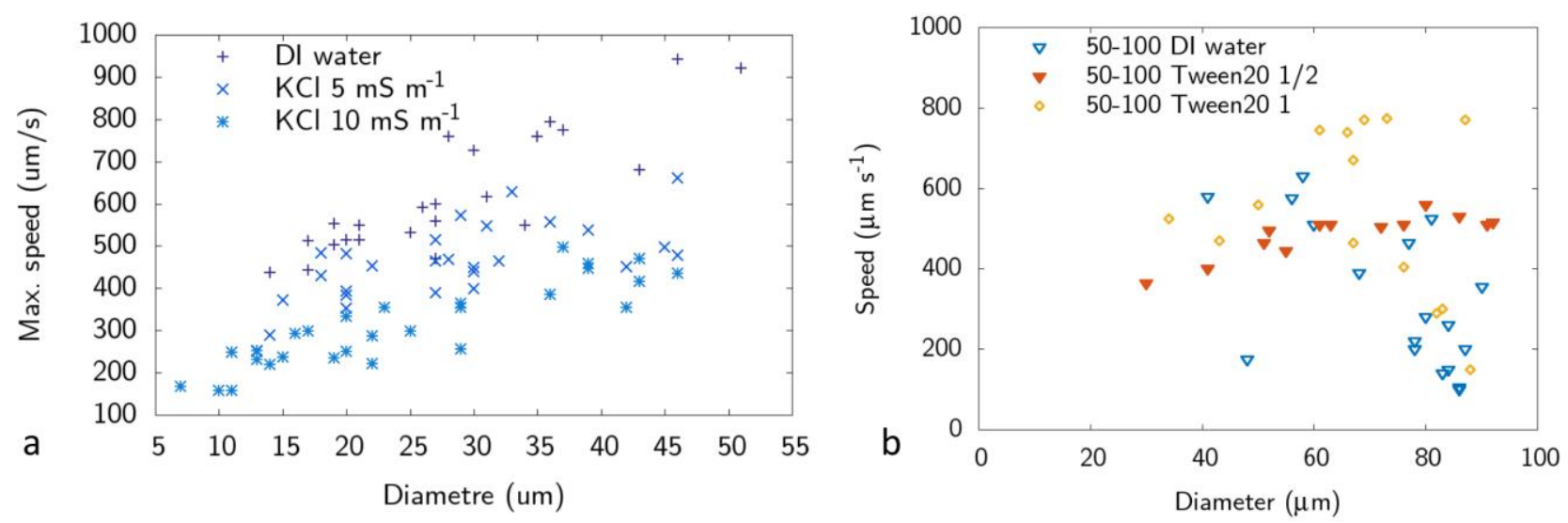

Figure 4 shows the results of the maximum speed at which we could spin glass beads of different sizes a) shows the results of smaller beads in different conductivity buffers and $b$ ) shows larger beads in buffers with different amounts of surfactant (Tween20).

Figure 4 shows the results of how fast we can move the beads of different sizes. We first conducted a set of experiments with smaller beads in three different buffers, DI water, DI water with potassium chloride to increase the conductivity to $5 \mathrm{mSm}^{-1}$ and $10 \mathrm{mSm}^{-1}$. It can be seen that the results in DI water produce the fastest manipulation speeds and that for low diameters the max speed increases roughly linearly with the size of the bead. However it can also be seen in figure 4a) that as the diameter of the bead increases to above $30 \mu \mathrm{m}$ the trend becomes less obvious and the results start to vary considerably. It is likely that in this second regime the mass of the beads starts to become important, and although as we have seen the viscous force still dominate over the inertial forces, the mass of the beads may be pushing them closer to the surface of the OET device where other frictional and stiction forces become important. Figure $4 \mathrm{~b}$ ) shows the results taken with larger beads and it can be seen that the speeds achievable in DI water start to decrease. As we believed that this decrease was due to the beads interacting with the surface we tried adding $1 / 2$ and 1 drop of Tween 20 , a non-ionic surfactant, to the buffer. Adding the Tween increased the speeds at which we could move these larger beads which backs up the hypothesis that it is the interaction with the surface which is reducing the speed at which they can be moved.

\section{CONCLUSION}

We have investigated the relationship between the speed at which we can move particles in an OET device and the size of the particle. We used a spinning wheel like light pattern that could be increased in speed until the beads no longer kept up with the light pattern and fell out of the trap. We have shown that the viscous drag force for these particles is larger than the centripetal forces and so the speed at which we can spin them is a good measure of our ability to move them against drag forces. We have found that the speed that particle can be moved at increases roughly linearly for beads under 30 microns in diameter but then starts to vary greatly as the mass of the beads starts to become important and their 
interactions with the surface start to have an effect. The maximum speed that we could move particles at was $943 \mu \mathrm{ms}^{-1}$ which was for a 46 micron diameter bead. We found that the maximum speeds achievable with beads of this size was vary variable and that for larger beads the maximum speeds started to decrease. We found that the addition of surfactants helped to increase the speed at which the larger beads could be moved giving more evidence that it is the interaction between the bead and the surface that causes the reduction in speed.

\section{ACKNOWLEDGEMENTS}

We would like to thank the staff of the James Watt Nanofabrication Centre (JWNC) for their help in fabricating the OET devices. This work was supported by a CONAYCT scholarship and associated visiting student travel grant.

\section{REFERENCES}

1. Chiou, P.Y., Ohta, A.T. and Wu, M.C., 2005. Massively parallel manipulation of single cells and microparticles using optical images. Nature, 436(7049), pp.370-372.

2. Hsu, H.Y., Ohta, A.T., Chiou, P.Y., Jamshidi, A., Neale, S.L. and Wu, M.C., 2010. Phototransistor-based optoelectronic tweezers for dynamic cell manipulation in cell culture media. Lab on a Chip, 10(2), pp.165-172.

3. Ohta, A.T., Chiou, P.Y., Han, T.H., Liao, J.C., Bhardwaj, U., McCabe, E.R., Yu, F., Sun, R. and Wu, M.C., 2007. Dynamic cell and microparticle control via optoelectronic tweezers. Microelectromechanical Systems, Journal of,16(3), pp.491-499.

4. Ohta, A.T., Garcia, M., Valley, J.K., Banie, L., Hsu, H.Y., Jamshidi, A., Neale, S.L., Lue, T. and Wu, M.C., 2010. Motile and non-motile sperm diagnostic manipulation using optoelectronic tweezers. Lab on a Chip,10(23), pp.3213-3217.

5. Ohta, A.T., Chiou, P.Y., Phan, H.L., Sherwood, S.W., Yang, J.M., Lau, A.N., Hsu, H.Y., Jamshidi, A. and Wu, M.C., 2007. Optically controlled cell discrimination and trapping using optoelectronic tweezers. Selected Topics in Quantum Electronics, IEEE Journal of, 13(2), pp.235-243.

6. Hwang, H., Lee, D.H., Choi, W. and Park, J.K., 2009. Enhanced discrimination of normal oocytes using optically induced pulling-up dielectrophoretic force. Biomicrofluidics, 3(1), p.014103.

7. Ohta, A.T., Jamshidi, A., Hsu, H.Y., Valley, J.K., Wu, M.C., Chiou, P.Y. and Neale, S.L., 2010. Optoelectronic Tweezers for the Manipulation of Cells, Microparticles, and Nanoparticles. INTECH Open Access Publisher.

8. Ohta, A.T., Neale, S.L., Hsu, H.Y., Valley, J.K. and Wu, M.C., 2008, August. Parallel assembly of nanowires using lateral-field optoelectronic tweezers. In 2008 IEEE/LEOS International Conference on Optical MEMS and Nanopotonics.

9. Neale, S.L. Ohta, A.T., Hsu, H.Y., Valley, J.K., Jamshidi, A., Wu, M.C., Trap profiles of projector based optoelectronic tweezers (OET) with HeLa cells, Optics Express, 17 (7): 5232-5239 (2009).

10. Pohl, H. A., Dielectrophoresis: the behaviour of neutral matter in nonuniform electric fields, Cambridge University Press, Cambridge (1978).

11. Neale, S.L., Wu, M.C., Trap stiffness in negative Optoelectronic Tweezers, Conference on Lasers and ElectroOptics (CLEO), DOI 10.1109/CLEO.2008.4551481 (2008).

12. Jones, T. B., Electromechanics of particles, Cambridge University Press, Cambridge (1995).

13. Neale, S.L., Mazilu, M., Wilson, J.I.B., Dholakia, K., Krauss, T.F., The resolution of optical traps created by light induced dielectrophoresis (LIDEP), Optics Express, 1:15(20): 12619-26.

14. Chiou, P.-Y., Ohta, A. T.., Wu, M. C., "Toward all optical lab-on-a-chip system: Optical manipulation of both microfluid and microscopic particles," Proc. SPIE - Int. Soc. Opt. Eng. 5514, 73-81 (2004).

15. Neale, S.L., Witte, C., Cooper J.M., Portable Optoelectronic Tweezers (OET), taking optical micromanipulation out of the optics lab, European Optical Society Annual Meeting (EOSAM) Aberdeen (2012). 